\author{
JOURNAL OF OBJECT TECHNOLOGY \\ Published by AITO - Association Internationale pour les Technologies Objets \\ http://www.jot.fm/
}

\title{
Introduction to the Meta'16 Special Issue
}

\author{
Elisa Gonzalez Boix ${ }^{\mathrm{a}}$
}

\author{
Stefan Marr ${ }^{b}$
}
a. Vrije Universiteit Brussel
b. University of Kent

This special issue represents a selection of the best papers of the workshop on Meta-Programming Techniques and Reflection 2016 (Meta'16). Meta'16 was held in Amsterdam, The Netherlands, in October 2016 co-located with SPLASH'16.

Meta is an ACM SIGPLAN workshop for discussing research on metaprogramming and reflection. The changing hardware and software landscape, and the increased heterogeneity of systems make metaprogramming once more an important research topic to handle the associate complexity. The scope of the workshop includes a wide range of topics related to design, implementation, and application of metaprogramming techniques, as well as empirical studies on and typing for such systems and languages.

The workshop welcomes mature contributions as well as work-in-progress contributions. A formal refereeing process selects a high-quality set of papers from those submitted at the workshop. Mature contributions are formally published in the workshop proceeding published electronically in the ACM Digital Library. The rest of papers are informally published at the workshop website. The JOT journal version of the papers offered an opportunity for the authors to take a longer term view of their research work and to present new results since the original presentation at Meta'16.

Meta'16 received 14 submissions, of which 7 full papers and 3 short papers were accepted and presented at the workshop. The guest editors of this special issue selected seven papers from Meta'16 workshop and invited their authors to submit an extended version of their paper, including at least 30\% of novel material. All papers have been reviewed by at least three reviewers, and have followed the reviewing process of the JOT journal until the final decision for each paper was reached.

After the journal's rigorous reviewing process, the editors of this special issue finally selected the following two papers for publication:

1. Yutaro Tsunekawa, Taichi Tomioka, Kazunori Ueda. Implementation of LMNtal Model Checkers: a Metaprogramming Approach. This paper discusses an approach for a meta-circular interpreter for prototyping model checkers.

2. Pablo Tesone, Guillermo Polito, Noury Bouraqadi, Stéphane Ducasse, Luc Fabresse. Dynamic Software Update from Development to Production. This paper discusses a software update solution suitable for live programming environments. 
As editors of this special issue, we hope you will enjoy the selection of papers. We would like to sincerely thank the Meta'16 program committee and the anonymous referees who provided extensive feedback on the submitted papers; their reviews helped both authors and us, guest editors, to improve the quality of the submissions. 